\title{
Oxygen and Light Elements Synthesis by Neutron-Capture Reactions in Metal-Free and Extremely Metal-Poor AGB Stars
}

\author{
Takanori Nishimura* \\ Astronomical Data Archives Center, National Astronomical Observatory of Japan \\ E-mail: nishimura.takanori@nao.ac.jp
}

Masayuki Aikawa

Hokkaido University OpenCourseWare, Hokkaido University

E-mail: aikawadec.hokudai.ac.jp

\section{Takuma Suda}

Department of Physics, Faculty of Science, Hokkaido University

E-mail: sudalastro1.sci.hokudai.ac.jp

\section{Masayuki Fujimoto}

Department of Physics, Faculty of Science, Hokkaido University

E-mail: fujimoto@astrol.sci.hokudai.ac.jp

The metal-free and extremely metal-poor stars of low- and intermediate-masses experience a mixing event of hydrogen into the convection driven by the helium shell flashes during early TP-AGB phase, differently from metal-rich stars. We study the nucleosyntesis in the helium convective zone with hydrogen mixing by computing the progress of nucleosynthesis with ${ }^{13} \mathrm{C}$ formed from the mixed proton as a neutron source by a nuclear network covering from hydrogen through sulfur. In the dearth of the pristine metals, the neutron-recycling reactions, ${ }^{12} \mathrm{C}(n, \gamma){ }^{13} \mathrm{C}(\alpha, n){ }^{16} \mathrm{O}$, and the subsequent ${ }^{16} \mathrm{O}(n, \gamma){ }^{17} \mathrm{O}(\alpha, n){ }^{20} \mathrm{Ne}$ reactions promote the syntheses of oxygen through magnesium and still heavier elements, including their neutron-rich isotopes and the odd atomic number elements. Our results are compared with the observations of the most iron-deficient, carbon-rich stars of HE 0107-5240 $([\mathrm{Fe} / \mathrm{H}]=-5.3)$, HE 1327-2326 $([\mathrm{Fe} / \mathrm{H}]=-5.4)$ and $\mathrm{HE}$ $0557-4840([\mathrm{Fe} / \mathrm{H}]=-4.75)$ to show that their peculiar abundance patterns of light elements can be reproduced in terms of the nucleosynthesis of metal-free AGB stars of different masses. These three stars were born as the low-mass members of Population III binaries and later subject to the surface pollution both by the mass transfer in the binary systems and by the accretion of interstellar gas. The other extremely metal-poor stars also have evolved from the binaries with low- and intermediate-mass primary stars.

10th Symposium on Nuclei in the Cosmos

July 27 - August 12008

Mackinac Island, Michigan, USA

\footnotetext{
* Speaker.
} 


\section{Introduction}

During the past decade, the studies of extremely metal-poor (EMP) stars in the Galactic halo with high resolution spectroscopy have revealed abundance patterns different from those known from previous observations and predicted from theoretical calculations. These stars can be a unique probe into the structure formation of early Universe. In order to grasp the origin of strange abundances we investigate the progress of nucleosynthesis in the He-flash convection taking into account the particular mechanism under metal poor situation. In this paper, we distinguish the two stars of $[\mathrm{Fe} / \mathrm{H}]<-5$ (HE0107-5240: [1] and HE1327-2326: [2]) and the other star of $[\mathrm{Fe} / \mathrm{H}]<-4$ (HE0557-4840: [3]) from other EMP stars by calling them as hyper metal-poor (HMP) and ultra metal-poor (UMP) stars, respectively, as proposed by [4].

\section{Special Mixing and Dredge-up in EMP Stars}

A distinctive feature of the evolution of EMP stars is the hydrogen mixing into the helium convection during the helium shell flashes. The carbon included in convection captures the mixed protons and produces the ${ }^{13} \mathrm{C}$ via ${ }^{12} \mathrm{C}(p, \gamma){ }^{13} \mathrm{~N}\left(e^{+} v\right){ }^{13} \mathrm{C}$ reactions. Being transfered to interior, ${ }^{13} \mathrm{C}$ makes a neutron with ${ }^{13} \mathrm{C}(\alpha, n){ }^{16} \mathrm{O}$ reactions at the middle of helium convective zone. The produced neutrons open paths to the nucleosynthesis which can reach the elements heavier than oxygen (see section 5). The nuclear products in helium zone are carried out to the surface by a couple of dredge-up mechanisms called He-flash driven deep mixing (He-FDDM) and third dredge up (TDU). He-FDDM occurs after the shell flash grows strong enough to engulf hydrogen at sufficiently high rate, and TDU might follows in some case depending on stellar mass.

\section{Nucleosynthesis}

We investigate the nucleosynthesis induced by above special mixing with nuclear network which includes isotopes and reaction rates from [5-9. We can construct the evolutions of temperature and density at the bottom of helium convection with use of analytical solution in the helium shell by [10]. To grasp the event related the special mixing we use ${ }^{13} \mathrm{C}$ supplied to helium shell instead of mixed protons. We can cover the various situations by treating mixed values and durations of ${ }^{13} \mathrm{C}$ as parameters and by considering some flash models.

\section{The Binary Scenario}

We compare the products abundances in EMP AGB nucleosynthesis with surface abundances of observed EMP stars from the viewpoint of the binary scenario [11]. The dredged up products in helium zone to the surface of erstwhile primary stars by He-FDDM and also by TDU, following He-FDDM in some case, planted on the low-mass secondary members through wind accretion to be observed as surface pollution. Since the yields may suffer dilution with the envelope matter both in convective envelope of primary stars when they are dredged up and in the surface convection of secondary stars when they are accreted, it makes sense to compare the relative abundances among the elements. 


\section{Oxygen production}

Figure. 1 shows the oxygen to carbon ratios against the carbon abundances among the observed EMP stars, with the theoretical predictions from the nucleosynthesis in AGB stars. According to theoretical approach there are two ${ }^{16} \mathrm{O}$ sources in AGB phase of EMP stars which have different $\mathrm{O} / \mathrm{C}$ production ranges. The one is ${ }^{12} \mathrm{C}(n, \gamma){ }^{13} \mathrm{C}(\alpha, n){ }^{16} \mathrm{O}$ with special hydrogen mixing, which can approach $[\mathrm{O} / \mathrm{C}]=-0.66$. The other is the case with no special hydrogen mixing which achieve $[\mathrm{O} / \mathrm{C}]=-2.4--1.65$ through ${ }^{12} \mathrm{C}(\alpha, \gamma){ }^{16} \mathrm{O}$ during TDU. In addition to above AGB nucleosynthesis "other EMP stars", which denotes the sub group of not-CEMP EMP stars $([\mathrm{C} / \mathrm{Fe}]<0.5)$ imply other oxygen source(s) which follow $[\mathrm{O} / \mathrm{Fe}]=0.5-1.0$. In other words, some oxygen has been accreted onto the primary/secondary star surface before the dredge-up/transfer events. The $\mathrm{O} / \mathrm{C}$ ratios of almost all EMP stars can be explained in terms of AGB nucleosynthesis during hydrogen mixing and/or during TDU with pristine oxygen abundances $[\mathrm{O} / \mathrm{Fe}]=0.5,1.0$.

\section{Constrains on $\mathrm{Mg} / \mathrm{Na}$ and $\mathrm{Al} / \mathrm{Mg}$ ratios}

Figure. 2 shows the $\mathrm{Mg} / \mathrm{Na}$ vs. $\mathrm{Al} / \mathrm{Mg}$ as well as Figure.1. The evolutionary paths imply that $\mathrm{Al}$ is produced via ${ }^{26} \mathrm{Mg}(n, \gamma){ }^{27} \mathrm{Al}$ reaction as $\mathrm{Mg}$ increases. Therefore the $\mathrm{Al} / \mathrm{Mg}$ ratio does not over the upper limit decided from the ratio of neutron capture cross sections. One more thing readable from evolutionary paths is that $\alpha$ capture reaction of ${ }^{22} \mathrm{Ne}(\alpha, \gamma)^{26} \mathrm{Mg}$ might be effective after neutron supply ended in case of high temperature flash. So the produced $\mathrm{Mg} / \mathrm{Na}$ ratio fall in righter than evolutionary paths. The two HMP stars lie near the evolution curves, on the other hand, the UMP star is in the same range as other EMP stars with no C-rich which imply pristine Mg.

\section{AGB nucleosynthesis in primary stars with different mass ranges can reproduce the abundance patterns on HMP/UMP stars}

In Figure. 3 the observed abundance patterns from HMP/UMP stars are compared with some of results from AGB nucleosynthesis in helium convection. These results will be affected by later He-FDDM or TDU in some cases. For example CN-cycle reactions attendant with dredge-up events will convert carbon to nitrogen, and TDU will dilute the envelope by dredged up carbon and oxygen. The HE1327-2326 pattern is reproduced by the results in helium convection except nitrogen. Since the nitrogen will be formed from carbon, we can explain the HE1327-2326 pattern by the products which are from nucleosynthesis with hydrogen mixing and are dredged up by He-FDDM. The HE0107-5240 pattern is explained by the products in helium convection diluted by carbon and oxygen associated with TDU. Finally, The HE0557-4840 pattern indicates carbon enhancement only and does not match in light elements with products in helium zone. This fact imply the TDU and strong contribution of pristine elements.

Hydrogen mixing and He-FDDM works for stars of mass $M \lesssim \sim 3 M_{\odot}$ until the CNO abundances in the envelope exceeds $[\mathrm{CNO} / \mathrm{Fe}] \gtrsim-2.5$ [12]13]. For stars of mass $M \gtrsim \sim 1.5 M_{\odot}$, TDU follows He-FDDM to enrich the envelope with ${ }^{12} \mathrm{C}$ and ${ }^{16} \mathrm{O}$ but without nitrogen. The three stars represent all the possible paths to carbon enrichment in the binary scenario; HE1327-2326 with the primary stars in the mass range $M_{p} \lesssim 1.5 M_{\odot}, \mathrm{HE0107-5240}$ in the mass range $1.5 M_{\odot} \lesssim M_{p} \lesssim 3 M_{\odot}$ and HE0557-4840 in the mass range $M_{p} \gtrsim 3 M_{\odot}$. 


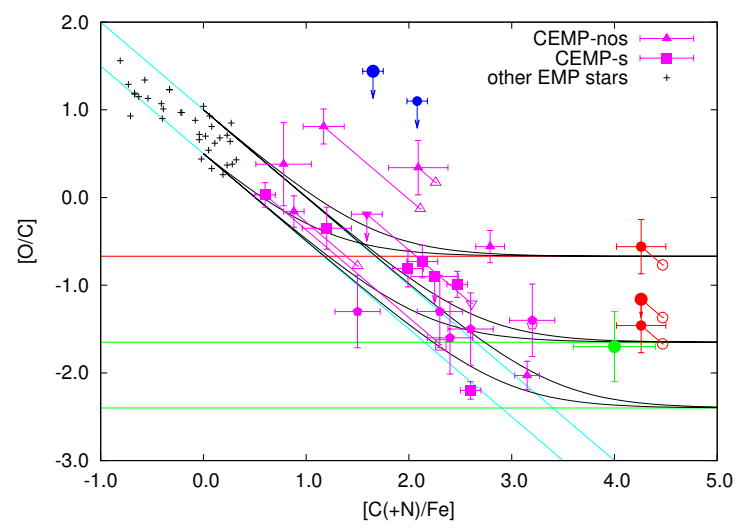

Figure 1: $[\mathrm{C}(+\mathrm{N}) / \mathrm{Fe}]$ vs. $[\mathrm{O} / \mathrm{C}]$ among observed EMP stars with theoretical predictions. For N-rich case we added the $\mathrm{N}$ abundances to $\mathrm{C}$ abundances explicitly because the nitrogen are formed from carbon via $\mathrm{CN}$ cycle reactions. The horizontal lines denote the upper bound from ${ }^{12} \mathrm{C}(n, \gamma){ }^{13} \mathrm{C}(\alpha, n){ }^{16} \mathrm{O}$ during hydrogen mixing and the range from ${ }^{12} \mathrm{C}(\alpha, \gamma){ }^{16} \mathrm{O}$ during TDU, respectively. Two oblique lines denote the pristine oxygen abundances of $[\mathrm{O} / \mathrm{Fe}]=0.5,1.0$. And the mixture of horizontal ranges and oblique abundances are indicated by curves.

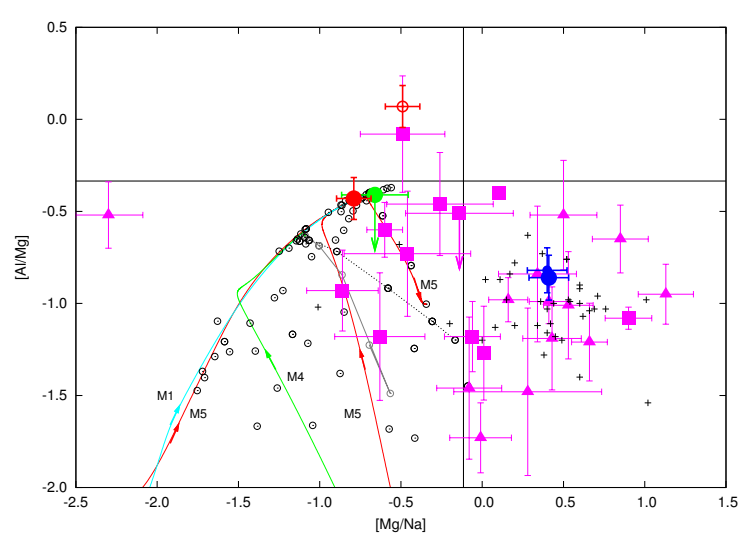

Figure 2: $[\mathrm{Mg} / \mathrm{Na}]$ vs. $[\mathrm{Al} / \mathrm{Mg}]$ among observed EMP stars and calculated results with theoretical constraints. Open circles are the calculated results. In some case the time evolutions are denoted by curves with arrows. The horizontal and vertical lines indicate the neutron capture upper bounds derived from the ratios of neutron capture cross sections.

The different abundance patterns among HMP/UMP stars are all explained by the binary scenario with different primary masses. Their tiny iron abundances are due to the later accretion of interstellar gas polluted by ejecta of population III and subsequent-generations of supernovae.

\section{References}

[1] Christlieb, N. et al. 2002, A stellar relic from the early Milky Way, Nature, 419, 904

[2] Frebel, A. et al. 2005, Nucleosynthetic signatures of the first stars, Nature, 434, 871

[3] Norris, J. E. et al. 2007, HE 0557-4840: Ultra-Metal-Poor and Carbon-Rich, ApJ, 670, 774 


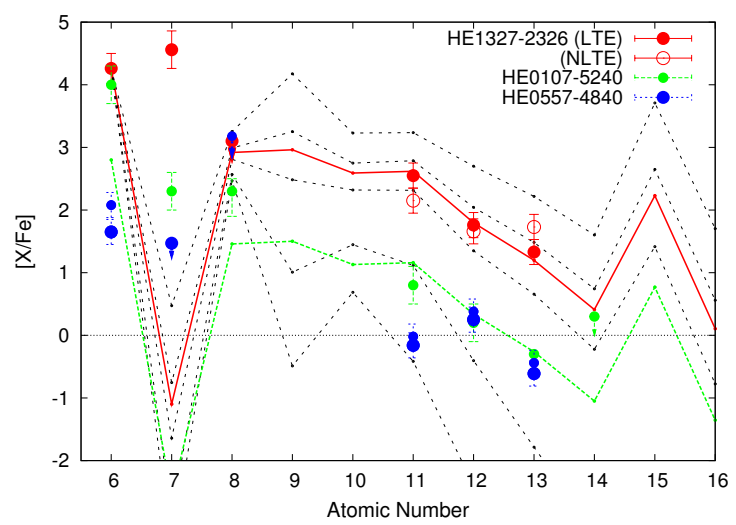

Figure 3: Comparison of the yields from the nucleosynthesis in the helium convection with enhancements of light elements observed for the three HMP/UMP stars. The observed data are taken from [14] for HE01075240, from [15] for HE1327-2326 and from [3] for HE0557-4840. The oxygen abundance for HE0107-5240 is taken from [16. The upper limit derived for [O I] at $\lambda 6300$ for HE1327-2326 is taken from [17].

[4] Beers, T. C. \& Christlieb, N. 2005, The Discovery and Analysis of Very Metal-Poor Stars in the Galaxy, ARAA, 43, 531

[5] Angulo, C. et al. 1999, A compilation of charged-particle induced thermonuclear reaction rates., Nuclear Physics A, 656, 3

[6] Caughlan, G. R. \& Fowler, W. A. 1988, Thermonuclear Reaction Rates V, ADNDT, 40, 283

[7] Bao, Z. Y. et al. 2000, Neutron Cross Sections for Nucleosynthesis Studies, ADNDT, 76, 70

[8] Koehler, P. E. \& Graff, S. M. 1991, ${ }^{17} \mathrm{O}(n, \alpha){ }^{14} \mathrm{C} C$ cross section from 25 meV to approximately 1 MeV, Physical Review C, 44, 2788

[9] Rauscher, T. \& Thielemann, F.-K. 2000, Astrophysical Reaction Rates From Statistical Model Calculations, ADNDT, 75, 1

[10] Sugimoto, D. \& Fujimoto, M. 1978, A General Theory for Thermal Pulses of Finite Amplitude in Nuclear Shell-Burnings, PASJ, 30, 467

[11] Suda, T. et al. 2004, Is HE 0107-5240 A Primordial Star? The Characteristics of Extremely Metal-Poor Carbon-Rich Stars, ApJ, 611, 476

[12] Fujimoto, M. Y., Ikeda, Y., \& Iben, Jr., I. 2000, The Origin of Extremely Metal-poor Carbon Stars and the Search for Population III, ApJL, 529, L25

[13] Suda, T. \& Fujimoto, M. Y. 2008, Stellar Evolution of Low-and Intermediate-Mass with [Fe/H] $\leq-3$, ApJ, submitted

[14] Christlieb, N. et al. 2004, HE 0107-5240, a Chemically Ancient Star. I. A Detailed Abundance Analysis, ApJ, 603, 708

[15] Aoki, W. et al. 2006, HE 1327-2326, an Unevolved Star with [Fe/H] <-5.0. I. A Comprehensive Abundance Analysis, ApJ, 639, 897

[16] Bessell, M. S., Christlieb, N., \& Gustafsson, B. 2004, On the Oxygen Abundance of HE 0107-5240, ApJL, 612, L61

[17] Frebel, A. et al. 2006, The Oxygen Abundance of HE 1327-2326, ApJL, 638, L17 